\title{
CERVANTES Y LOS JESUITAS
}

Todos los biógrafos modernos de Cervantes o editores de las Novelas ejemplares se han referido con variada extensión a su posible asistencia entre los 7 y los 17 años (1554-1564) a las escuelas de gramática y humanidades que habían abierto por esas fechas los jesuitas en Córdoba y Sevilla ${ }^{1}$. Ante la carencia de un testimonio fehaciente en un sentido o en otro, el fundamento de esa posibilidad ha sido y sigue siendo el conocido elogio de tales escuelas, que Cervantes pone en boca de Berganza y Cipión en Novela y coloquio [de los perros], una de las Ejemplares publicadas en 1613 y escrita probablemente no antes de 1604 . El carácter indirecto de la prueba no autoriza a reforzarla o minimizarla con interpretaciones globales de las intenciones y juicios latentes del escritor sobre la sociedad en la que vivía y sobre la orden religiosa que en ella actuaba; menos aún con las opiniones personales del historiador sobre ambos conjuntos.

Es necesario por consiguiente separar el posible dato biográfico del contacto de Cervantes con los jesuitas, de la apreciación positiva o negativa que el gran observador de la realidad hispana de su tiempo pudo conservar de esa experiencia. Fuera de los años de la primera juventud, no se ha sugerido que pudiera haber tenido otros. Sin embargo conviene recordar que Cervantes estuvo en la cárcel real de Sevilla en 1597-abril 1598, sin contar otra posible estancia

1 Una excepción es Amelia Agostini de Del Río, Compañero del estudiante del Quijote (San Juan, Puerto Rico, 1975), que en la «Biografía de Cervantes» (pp. 27ss) omite ambas circunstancias. Los editores de la Obra Completa (Alcalá de Henares, 1993, I, xi) tienen por "cosa muy hipotética» los cursos con los jesuitas en Valladolid, Córdoba o Sevilla. 
en la ciudad en 1602-03. Y de la cárcel era capellán desde 1578 el jesuita Pedro de León (n.1545), alumno a su vez de los jesuitas de Sevilla en 1563-65, es decir, cuando se supone que pudo serlo Cervantes. Entre 1589 y 1603 recorrió o residió en diversas localidades andaluzas. Es muy improbable que no se hayan encontrado alguna vez ${ }^{2}$.

Por mi parte, deseo fijarme ante todo en esa posible peripecia biográfica. Dicho de otro modo más general: ¿qué clase de contactos pudo tener Cervantes con los jesuitas? Cualesquiera que fuesen sus ocultas reservas en la edad madura, si las tuvo, ¿pudo escribir ese elogio desde el simple conocimiento de la existencia de una orden religiosa, generalmente apreciada por su actividad docente? ¿Le bastaba para hacerlo con lo que había oído hablar de ellos a lo largo de su vida?

Puesto que nuestra base de indagación es un texto, convendrá tenerlo presente en sus afirmaciones principales (en cursiva, los conceptos que me parecen especialmente significativos):

BERGANZA.-[...] Este mercader tenía dos hijos, el uno de doce años y el otro de hasta catorce años, los cuales estudiaban gramática en el estudio de la Compañía de Jesús; iban con autoridad, con ayo y con pajes que les llevaban los libros y aquel que llaman «vademecum». El verlos ir con tanto aparato [...].

CIPIón.-[...] Sigue tu cuento, que le dejaste en la autoridad con que los hijos del mercader tu amo iban al estudio de la Compañía de Jesús.

BERGANZA.-A él me encomiendo en todo acontecimiento. [...]. No sé qué tiene la virtud, que, con alcanzárseme a mí tan poco o nada della, luego recibí gusto de ver el amor, el término, la solicitud y la industria con que aquellos benditos padres y maestros enseñaban a aquellos niños, enderezando las tiernas varas de su juventud, porque no torciesen ni tomasen mal siniestro en el camino de la virtud, que juntamente con las letras les mostraban. Consideraba cómo los reñían con suavidad, los castigaban con misericordia, los animaban con ejemplos, los incitaban con premios, y los sobrellevaban con cordura, y finalmente, cómo les pintaban la fealdad y horror de los vicios, y les dibujaban la hermosura de las virtudes, para que, aborrecidos ellos y amadas ellas, consiguiesen el fin para que fueron criados.

CIPIÓN.-Muy bien dices, Berganza; porque yo he oído decir desa bendita gente que para repúblicos del mundo, no los hay tan prudentes en todo él, y para guiadores y adalides del camino del cielo, pocos les llegan. Son espejos donde se mira la honestidad, la católica doctrina, la singular prudencia y, finalmente, la humildad profunda, base sobre quien se levanta todo el edificio de la eterna bienaventuranza.

BERGANZA.-Todo es así como dices. [...] Es el caso que a aquellos señores maestros les pareció que la media hora que hay de lición a lición la

2 Cf. las memorias del P. LEÓN, Grandeza y miseria en Andalucía, ed. P. HERRERA PUGA. Granada, 1981, 193-600. Del mismo editor, Sociedad y delincuencia en el Siglo de Oro. Madrid, 1974. 
ocupaban los estudiantes no en repasar las liciones, sino en holgarse conmigo; y así ordenaron a mis amos que no me llevasen más al estudio: obedecieron [...].

Antes de cualquier razonamiento, fijemos los jalones cronológicos de esos diez años cervantinos.

La presencia de Rodrigo de Cervantes en Córdoba consta por un documento del 30 de octubre de 1553, publicado por Astrana Marín ${ }^{3}$. Después de la bancarrota de Valladolid, con embargo de todas sus pertenencias y encarcelamiento por insolvencia (todo ello ampliamente documentado por Astrana), Rodrigo no podía continuar en la ciudad del Pisuerga; volvió con toda su familia a Alcalá a principios del verano de 1553, «para cobrar la renta del pan cogido" en las escasas tierras que le quedaban. Astrana encuentra natural que después buscase refugio con sus padres en Córdoba, en donde había vivido de niño. ¿Le siguió su familia? Werner Krauss supone gratuitamente que Miguel quedó en Alcalá con su tía Ma$\mathrm{ría}^{4}$, y da por única razón que esas separaciones eran entonces frecuentes. ¿Por qué iba a quedar, cuando María de Cervantes había vendido con autorización de su padre la casa familiar, y tenía que vivir con su hija natural casada? Pero además, el documento firmado por Rodrigo en octubre de 1553 es una escritura de obligación por valor de 4.660 maravedís, «por doce varas de ruán y dieciocho varas más un tercio de holanda», a pagar en la Navidad de 1554. ¿Para qué las podría necesitar, sino para reponer el ajuar y vestir a su mujer y cinco hijos, que habían salido de Valladolid con lo puesto? ${ }^{5}$

Astrana ha reconstruido con un «criterio de razón, [que] es, a veces, más seguro que el documental», los años de la primera escolaridad de Miguel: primero (1553) en la escuela primaria del clé-

3 Vida ejemplar y heroica, I, 286. M. Herrero García, en su Vida de Cervantes (Madrid, 1948), y luego en "Casos cervantinos que tocan a Madrid" (RBAMMadrid, 1951, 7-12), sostiene que la familia Cervantes residió en Valladolid ininterrumpidamente hasta su traslado a Madrid en 1561, y consiguientemente el discipulado con los jesuitas habría tenido lugar en la primera. Esto es del todo improbable, como se verá en la n.5.

4 "Cervantes und die Jesuiten in Sevilla", Romanische Forschungen, 54, 1940 391; y lo repite en Miguel de Cervantes, Leben und Werk. Berlín, 1966. Siguiendo a Rodríguez Marín, ignora la etapa cordobesa anterior. Sorprende que Bataillon persista en la misma ignorancia, y considere fundada la hipótesis de Krauss (Erasme et l'Espagne [Génève, 1991] II, 329s).

5 Los bienes embargados a Rodrigo en 4 de julio de 1552 incluyen mantas, "cuatro sábanas», varias almohadas de cama, "servilletas de mesa», "dos sábanas y una tabla de manteles», "dos toballetas de lienzo» y "dos sábanas de ruán» (Astrana, 269s.). ¿Tiene sentido comprarlo en Córdoba para llevarlo a Valladolid? Coincido con C. Zaragoza, Cervantes, vida y semblanza. Madrid, 1991, 52; el documento en p. 378 . 
rigo Alonso de Vieras ${ }^{6}$, posible pariente de los Cervantes, como hijo de un Gonzalo de Cervantes, de Granada. Alonso de Vieras tenía dos sobrinos carnales, Gonzalo de Cervantes Saavedra y Alonso de Cervantes Sotomayor. Otros cordobeses coetáneos serán elogiados más tarde por Miguel o incluirán elogios de éste en los preliminares de sus obras, lo que podría interpretarse como indicio de antigua amistad ${ }^{7}$.

Sobre la fundación de los jesuitas en Córdoba y el comienzo de las escuelas en diciembre de 1553, resume Astrana los datos del historiador Astrain ${ }^{8}$. Añade un importante documento del 16 de enero de 1554, por el que el fundador y deán del cabildo, D. Juan de Córdoba, asienta definitivamente las condiciones de la fundación: uno de los testigos es «el licenciado Cervantes», es decir, el abuelo de Miguel $^{9}$.

Astrana acepta la lectura que había hecho Rodríguez Marín ${ }^{10}$ del pasaje del Coloquio como prueba del discipulado, pero la corrige al situar la acción años antes en Córdoba. Ni el mercader sevillano, padre de los escolares, puesto en ridículo por el escritor, pudo ser Rodrigo de Cervantes; ni la Lonja, en la que durante las clases de éstos negociaba, se abrió antes de 1598. Cervantes aprovecharía la circunstancia sevillana, que le era tan familiar, para fundir en uno todos sus recuerdos.

Muerto el licenciado Juan de Cervantes en 1556 y su mujer Leonor de Torreblanca en 1557 (después de vender «un esclavo de color loro» en el precio de setenta ducados, indicio de las estrecheces que de nuevo atenazaban a la familia), Rodrigo con su familia abandonó Córdoba para trasladarse a la vecina Cabra, donde vivía acomodadamente su hermano Andrés. No se vuelve a tener noticia

6 R. Aguilar PRIEgo, «Maestros cordobeses de primeras letras, siglos xv al xviii", Boletín de la R. Academia de Córdoba, 76, 1959, 123-140: reproduce los datos de Astrana.

7 Juan Rufo, elogiado en el Canto de Calíope, del l. VI de La Galatea, incluye un soneto en su poema La Austriada; Juan de Aguayo y Castilla, del que volveremos a hablar, es también elogiado en el Canto de Caliope, lo mismo que Gonzalo de Cervantes.

8 Historia de la CJ en la Asistencia de España, I, c. XII.

9 Astrana, I, 322-325. En el Archivo Romano de la Compañía de Jesús hay copia de otro documento, fechado el 24 de enero del mismo año, que parece ampliar y precisar el anterior. Hace efectiva la entrega prometida de trescientos libros, y promete todos los de su librería al tiempo de su muerte. En él no figura como testigo Juan de Cervantes (ARSI, FG, 1404-Córdoba). KrzYSZToF SLIWA-DANIEL EISEnBerG, "El licenciado Juan de Cervantes, abuelo de Miguel de Cervantes Saavedra", Cervantes, 17, 1997, 106-114, se refieren a la posible influencia que pudo ejercer sobre su nieto Miguel en esos años cordobeses (1553-56).

10 Cervantes estudió en Sevilla (Sevilla, 1915), reproducido en Estudios Cervantinos. Madrid, 1947, 51-64. 
de ellos hasta el 30 de octubre de 1564, cuando Rodrigo aparece en Sevilla como subarrendador de un cierto Juan Mateo de Ureña, que le debía la renta de tres meses. Astrana (p.399) conjetura que podrían haber llegado el año anterior, pero no dice en qué se funda. En la p.415 se refiere al curso 1563-64, en el que Miguel cursaría $3{ }^{\circ}$ de gramática. Con la familia de Rodrigo vivía Juan, hijo de su hermano Andrés.

Leamos ahora atentamente el texto del Coloquio. Por dos veces, primero en boca de Berganza y luego de Cipión, se refiere el escritor al estudio de la Compañía de Jesús. Y esto es ya una sorpresa para el lector: oir a unos canes - de quienes cabría esperar un lenguaje más próximo al coloquial del vulgo- expresarse con tal precisión canónica. Es la única referencia que encontramos en Cervantes sobre los jesuitas ${ }^{11}$; y no los llama «teatinos», como eran popularmente conocidos, no sólo entre el vulgo: así se refieren a ellos lo mismo Sta. Teresa que Fr. Melchor Cano y Fr. Domingo Báñez, o entre los profesionales de la pluma, Lope de Vega, Calderón, Quevedo, Tirso y Góngora (los tres primeros ciertamente alumnos suyos). La precisión, diríamos que puntillosa, no parece irrelevante ${ }^{12}$ : ¿proximidad afectiva, distanciamiento crítico, o más simplemente, homenaje oportuno para cubrirse ante posibles censuras por otras audacias de las novelas? El indicio es liviano para cualquier conclusión.

Antes de pasar al elogio, importa insistir sobre un prejuicio muy arraigado, que sirve a varios comentaristas de este episodio para negar verosimilitud al discipulado de Cervantes: el de la elevada condición social de los alumnos de los jesuitas. De que los hijos del mercader sevillano fueran al estudio "con autoridad de ayo y pajes» y "con aparato de sillas o coche», no se puede deducir que el impecunioso Rodrigo de Cervantes no pudiese enviar «a su hijo Miguel a un colegio de tan exclusiva dedicación a los retoños de la

11 CARlos Fernández Gómez, Vocabulario de Cervantes. Madrid, 1962.

12 Más natural y suficiente sería "estudio de la Compañía». Nótese que tanto en la edición princeps de Madrid (1613), como en las de Pamplona (1614 y 1622), Bruselas (1614 y 1625), Milán (1615) y Madrid (1622), por lo menos, se imprime «Compañía de IESVS». No se mantiene en la ed. de la Obra Completa (Alcalá, 1994), que se anuncia fiel al original. Vital D'Avdigvier (París, 1614) traduce: «lesquels estudioient/alloient aux Jesuites»; con lo que suprime el devoto propósito de Berganza: "A él (Jesús) me encomiendo en todo acontecimiento». ( ¿Una reminiscencia, en clave humorística, de la educación recibida?). D'Audiguier reprocha a Cervantes su tendencia a mezclar las cosas sagradas con las profanas: G. HAINSWORTH, Les "Novelas Exemplares" en France au XVII siècle, 1933, 61, n. 4. La misma sustitución hace Guglielmo Alessandro DE Nouilieri, Il Novelliere Castigliano. Venezia, 1629, p.653. B. W. IFE, Exemplary Novels IV, 1992, 101, traduce la primera vez por "Jesuit school" y la segunda, "the school run by the Jesuits"; a pesar de lo cual continúa: "I commended myself to Him». 
nobleza y de los poderosos mercaderes locales» ${ }^{13}$. El letrero que había aparecido el 11 de febrero de 1551 sobre la puerta de una modestísima casa (en la actual Via del Aracoeli, cercana al Campidoglio de Roma) decía escuetamente: "Scuola di grammatica, d'umanità e dottrina cristiana gratis». En el carácter de gratuidad insisten constantemente, tanto las Constituciones de la Orden como las de las universidades y colegios fundados en años siguientes ${ }^{14}$. Esto permitía una efectiva educación igualitaria ${ }^{15}$, de modo que los hijos del conde de Monterrey, fundador del colegio en la minúscula cabeza de sus estados, se codeaban en las clases con los centenares de muchachos que venían diariamente a pie desde dos leguas a la redonda, sostenidos con pan de centeno y agua ${ }^{16}$. Esta era la realidad del tiempo, y a ella hay que atenerse.

La primera parte del elogio, puesto en boca de Berganza, concluye con esta frase: consiguiesen el fin para que fueron criados. La expresión no pasará inadvertida para cualquier familiarizado con los escritos ignacianos ${ }^{17}$. Efectivamente, en sus Ejercicios espirituales y como pieza característica y en cierto modo emblemática - aunque en realidad sólo sea el pórtico del librito-, destaca la titulada «Principio y fundamento», que comienza: «El hombre es criado para

13 J. M. Caballero Bonald, Sevilla en tiempos de Cervantes. Barcelona, 1991, 122. "Luego, los estudiantes ricos van con los jesuitas, los cuales son con ellos infinitamente tiernos...» (MARía L. JAROCKA, El coloquio de los perros a una nueva luz, UNAM, México, 1979, 87). La misma idea en J. CANAVAGgio, Cervantes, en busca del perfil perdido. Madrid, 1992, 40.

14 El secretario del General, Juan de Polanco, escribe en 1551: "y habiéndose publicado esta escuela, admítense en ella gratis y sin accetar dinero ni presente alguno todos quantos quieren, que sepan leer y scrivir y comiencen la gramática...; que los pobres, que no tienen con qué pagar los maestros ordinarios, ni menos los pedagogos domésticos, aquí hallan gratis lo que con costa mucha apenas podrían aver para salir con las letras» (Monumenta Paedagogica S.I., I [1540-56] 415, 417. Otros textos en 60, 88, 307, 435s; VII [1598-1616] 305, 321, 333, 447, 465).

15 Si fueron escasos los convictorios o internados, se debió, aparte los problemas de administración, al deseo de prevenir las servidumbres que traerían ciertos alumnos.

16 E. Rivera VÁzquez, Galicia y los jesuitas. A Coruña,1989, 142, 176. Igual experiencia vivían en Monforte los tres hijos del VI Conde de Lemos, bisnietos por línea materna del P. Francisco de Borja y sobrinos del conde, luego duque de Lerma (ibid., pp. 322s; Cuad Est Gallegos, 15, 1960, 105-110, y 17, 1963, 245s). En 1570, desde el colegio de Sevilla se informaba que, al duplicarse los 300 alumnos de 1561, «por estar las clases tan llenas de gente, algunos principales y aun devotos, ponían sus hijos en otros estudios; y ansí, nuestras clases se enchían de pobres y rudos". Diríase que la perspectiva era la inversa. Convendrá equilibrarla con lo que otro informador dice en 1572 a propósito de los 700 estudiantes de ese momento, «la juventud más florida y más ilustre de la ciudad». (Mon. Paed., III, 482 y 511). Sobre el tema, ver B. BARTOLOMÉ, Historia de la acción educadora de la Iglesia en España, I. Madrid, 1995, 657-659.

17 La señala M. HERrero, Vida, 53. Lo pasa por alto M. GAscón, «Los jesuitas según Cervantes», BBMP, 23 (1947), 179-211. 
alabar, hacer reverencia y servir a Dios...», y concluye precisamente con este epifonema: «solamente deseando y eligiendo lo que más conduce para el fin que somos criados» ${ }^{18}$. La coincidencia no parece casual, aunque la idea pertenezca evidentemente al fondo común cristiano; y la posición conclusiva del epifonema hace más evidente la dependencia literaria.

Pero nadie parece haberse preguntado cómo llegó esta doctrina - con esta expresión - a conocimiento de Cervantes. Creemos que no pudo encontrarla en ningún libro castellano. El texto latino de los Ejercicios ignacianos se publicó en Roma, 1548, con reediciones en Coimbra (1553), Viena (1563), Burgos (1574), Sevilla (1587) y Valencia (1599). La frase en cuestión se traduce así: «sed consentaneum est ex omnibus ea demum, quae ad finem ducunt, eligere ac desiderare». Este texto latino fue utilizado por todos los directores de Ejercicios, aun en España, y por los redactores de las apologías y defensas que de ellos se hicieron; aun en las cartas privadas a Roma siempre se citan en latín ${ }^{19}$. Naturalmente, entre los jesuitas españoles circulaban copias manuscritas del original, de las que no todas procedían del mismo arquetipo; y precisamente, para prevenir la difusión de un texto más o menos corrupto y por la razón práctica de los que no sabían latín, se pidió insistentemente a los Padres Generales la edición del original castellano ${ }^{20}$. De Roma llegó siempre una negativa decidida, por las razones que apunta Iparraguirre. El hecho es que la primera edición es de 1615, y aun entonces en Roma.

¿Habría encontrado el texto en alguno de los escritores jesuitas contemporáneos? En 1583 se publicó la traducción castellana de $\mathrm{La}$ Vida del Padre Ignacio de Loyola, de Pedro de Rivadeneira; el libro III concluye con un largo c. 24, «De los colegios que tiene la Compañía para enseñar»: no hay en él nada que pueda relacionarse literalmente con nuestro texto. El conocido catequista Gaspar Astete

18 Ignacio emplea exclusivamente las formas "Criador», "criado», "criatura» (Concordancias ignacianas, Bilbao-Santander, 1996). Sobre el significado de "criados» en este texto, ningún comentarista ha pensado en cosa distinta del 'acto creativo de Dios'; pero D'Audiguier traduce: «ils parvinssent a la fin pour laquelle ils estoient nourris». Sorprende que Jean Cassou lo mantenga, sin otra modificación que "aux fins» (CERvantes, Nouvelles Exemplaires. Bibliothèque de la Pléiade [1956] 1488). La idea y su expresión, conjugada con otra muy conocida de las Confesiones agustinianas, se repite en el Persiles: "nuestras almas están siempre en continuo movimiento, y no pueden parar ni sosegar sino en su centro, que es Dios, para quien fueron criadas» (III, c. 1, y Confesiones I, 1). La consideración de Dios, centro del alma, ya se encuentra en La Galatea, II,61, y se repite en Persiles, IV,10. Véase A. G. DE AMEZÚA, Cervantes, creador de la novela corta española [1956], I,114.

19 I. IPARRAguirre, Práctica de los Ejercicios [1522-1556]. Bilbao-Roma, 1946, 157-163. No se ha señalado una fuente castellana para ese texto ignaciano.

20 I. IPARRAgUIRre, Historia de los Ejercicios, II, 352-354. 
publicó (Burgos, 1592) una Institución y Guía de la juventud cristiana $^{21}$ : no hace ni siquiera mención de esa página de los Ejercicios. Igual silencio en Tomás de Villacastín, que publica en Valladolid (1612; la primera ed., desconocida) su Manual de consideraciones y exercicios espirituales para saber tener oración mental; y en Pedro Sánchez, Libro del Reyno de Dios y del camino por do se alcanza (Madrid, 1599). Más sorprendente es el caso del célebre escritor vallisoletano Luis de la Puente, que publica en 1605 y en Valladolid su primera y magistral obra Meditaciones de los misterios de nuestra santa Fe con la práctica de la oración mental sobre ellos. En la Introducción se refiere a las fuentes en las que bebe su doctrina, que son la Sagrada Escritura, los santos Padres y los Ejercicios ignacianos, de los que hace un amplio elogio. La primera parte se abre con una «Meditación primera fundamental, del fin para el que fue criado el hombre y las demás cosas que le sirven» (p.54-62). Y a continuación: «Esta primera meditación es principio y fundamento de la vida espiritual». Pero no se formula el texto que estamos buscando, aunque se expongan evidentemente los mismos conceptos.

Una excepción parcial es Jerónimo de Ripalda, que en su famoso Catecismo (Burgos, 1591), al tratar de las obligaciones del cristiano, responde: «a buscar el fin último para que fue criado». Aunque la referencia a los Ejercicios es clara, la brevedad de la respuesta no tiene la fuerza del original. Si lo leyó Cervantes, tanto como fuente directa pudo servirle como despertador de un antiguo recuerdo.

Creo que esta indagación inductiva permite concluir con probabilidad próxima a la certeza moral, que Cervantes no encontró en ningún libro ese epifonema conclusivo. Sólo queda, por consiguiente, suponer que lo recibió por tradición oral, por haberlo oído, no una vez por casualidad ${ }^{22}$, sino por repetida asistencia a predicaciones de jesuitas, o por emplear una fórmula corriente entre los jesuitas del tiempo, por haber "hecho algunos ejercicios», los llamados de "primera semana», entre los que figuraba como pieza clave la consideración del fin del hombre y del mundo. Desde Córdoba precisamente se escribía en septiembre de 1555: "De pocos días a esta parte se junta buena copia de estudiantes con algunos que vienen

21 In- $8 .^{\circ}$ de 267 fol, concebido como «manual y pequeño y de poco precio: porque lo puedan leer los niños en sus escuelas, y los estudiantes en sus estudios, y cualquiera persona lo pueda traer consigo».

22 No creo que valga la pena ergotizar sobre la «portentosa memoria de Cervantes», capaz de recordar al "caballero Fonseca», simplemente enumerado una sola vez en el c. CXXXII del Tirante el Blanco (M. PELAyo, Orígenes de la novela, I, 393). (Otra explicación más obvia en W. T. MCCrEADY, $M L N, 73,1958,33-35$ ). 
de fuera, y tienen su hora de oración en la capilla; propóneles un Padre materia sobre qué mediten» ${ }^{23}$.

La perspectiva de un posible y prolongado contacto espiritual con los jesuitas, de lo que sería indicio ese leitmotiv recordado, viene a reforzar los argumentos recogidos por Astrana Marín sobre el discipulado de Cervantes en Córdoba y Sevilla. Pero ello ha dado pie a biógrafos y editores para especular sobre lo que el Cervantes maduro y cargado con las experiencias de su prodigiosa vida, podría haber pensado sobre los jesuitas y su actividad educativa.

Las interpretaciones modernas las sitúa Américo Castro, (después de censurar a las "gentes poco equilibradas...que [han] tratado de leer en [Cervantes] no sólo entre líneas, sino al trasluz») entre el «sincero elogio» y «una de tantas fórmulas de obsecuencia a las ideas oficiales». Concluye poniendo «a Cervantes de acuerdo con su tiempo e [intentando] ver las cosas un poco como él podía verlas», con lo que llega «a resultados tan distantes de la ingenuidad como de la insensata fantasía» ${ }^{24}$.

La lectura de unos y otros, desde Rodríguez Marín a Stanislav Zimic (para señalar extremos cronológicos, no valorativos), sugiere algunas observaciones, que pretenden mantenerse en el terreno de los datos objetivos y comprobables, lejos de los extremos señalados por Américo Castro. Lo que creo no se puede afirmar absolutamente es que «Cervantes siempre dijo con toda exactitud lo que quería decir» ${ }^{25}$. (¿También sobre los jesuitas?).

Que las alabanzas de Cervantes a los jesuitas sean «llamativamente hiperbólicas», es una primera impresión que, como a S. Zi-

23 MHSI, Litterae Quadrimestrae III, 627. En un Directorio latino para diversas clases de ejercitantes, redactado muy probablemente por el $4 .^{\circ}$ General, Everardo Mercurian (1573-1580), se dice: «si son niños, es decir, de siete a doce años, como muchos de nuestros alumnos que se confiesan cada mes, sus profesores, al menos una vez o dos por semana, pueden darles algunos (Ejercicios) segun su capacidad, no a modo de meditación, pues no sabrían meditar, sino a modo de alguna sentencia, para que la recuerden con frecuencia; o por la mañana durante breve rato, algo así como veinte o diez minutos, pues no pueden los niños mantener fija la imaginación por más tiempo». Y entre los temas que se sugieren, se apunta "fundamento del último fin». En el texto latino (MHSI, 76, 1955, 254s) se lee: «vel matutino tempore, per aliquam partem temporis, puta decimam vel vigesimam»; los editores creen que se debe suplir "partem horae» - lo que equivaldría a seis o tres minutos-, pero parece que hay que entenderlo del «tiempo escolar matutino", que para las clases inferiores era de 3,30 h. (R. G. Villoslada, Storia del Collegio Romano. Roma, 1954, 85s).

24 En este extremo se situaría "la imagen inversa de una denuncia feroz de los compromisos mundanos de la Orden» (J. CANAVAGGIO, o. c.).

25 MARJA L. JAROCKA, o. $c ., 126$. Sobre los prejuicios ideológicos de la autora, que falsean lamentablemente sus conclusiones, ver St. ZIMIC, Las Novelas Ejemplares. México-España, 1996, 338, n.15. 
mic $^{26}$, asalta a cualquier lector imparcial. Menos evidente es la apariencia de «obligación ritual, cumplida mecánicamente y abandonada cuanto antes» (¿cuál sería la forma y extensión óptimas? ¿Llegaría a las «impertinentes digresiones», que le reprocha Cipión a su compañero? Pero aquel añade por su cuenta una segunda parte, con riesgo evidente de «parecer predicadores»...). Menos claras aún las «flagrantes, estridentes discrepancias», que señala el mismo comentador. ¿Por qué no distinguir más equitativamente entre la «ostentación y aparato» del mercader, orgulloso de su éxito económico y social; el trabajo de los educadores con esas «tiernas varas», todavía no torcidas irremediablemente; y el juicio global del genial espectador de tan flagrante contraste? ¿Serían los educadores de los hijos responsables de la mentalidad y conducta de nouveaux riches de sus padres? ¿Tendrían que limitarse a los hijos de cristianos ejemplares? Que los amos de Berganza lo mantengan alejado del estudio, por orden de los maestros, no permite deducir que serían igualmente obedecidos éstos si pretendieran reformar el tren de vida de aquellos. La supresión de la inocente y alegre diversión de los colegiales con Berganza, en contrapunto a las monótonas rutinas de clase, impuesta por "una señora que llaman por ahí razón de estado", es posible, como piensa Zimic, que dé pie a "sutiles, atrevidas referencias críticas» del escritor ${ }^{27}$; aquel régimen escolar, que por esos años se ensayaba con lógicos tanteos en los diversos colegios jesuíticos ${ }^{28}$, podía ser duro, tanto para los discípulos como sobre todo para los maestros ${ }^{29}$; y la «erudición secamente libresca, rutinaria, irrelevante para la realidad humana a que debiera, ante todo, aplicarse», pudo ser una tacha de aquella pedagogía, de hecho generalmente aceptada y estimada; pero ¿habrá que medirla con los patrones del siglo XX (supuesto que alguno sea perfecto)? A otro propósito habló M. Pelayo peyorativamente de la «retórica de colegio»; pero ¿ «el ser latinos» predisponía a «ser asnos»? Más avisado era Berganza, cuando dio en repasar algunos latines «de muchos que oí cuando fui con mis amos al estudio, con que a mi parecer me hallé algo más mejorado de entendimiento».

26 O. c., p. 338, y antes en BBMPelayo, 1994, 71.

27 A. K. Forcione lo ve como "a joke», que apunta a las conocidas controversias de teoría política en las que intervenían los jesuitas por aquellos años (Cervantes and the Mystery of Lawlessness. Princeton, 1984, 153, n. 14). No es fácil encontrar un "tercio de comparación" para tan sutiles referencias.

28 Véanse abundantes pruebas en los siete tomos de Monumenta paedagogica S.I. Roma, 1965-1992. Notemos que la media hora entre clase y clase era una peculiaridad de la Ratio studiorum.

${ }_{29}$ El alto índice de fatigados, y aun de fallecidos, que se deduce de las páginas de Monumenta, lo prueba. 
Lo cierto es que Cervantes no dice una palabra, al menos directamente, de la calidad literaria de la enseñanza en los colegios. Descartemos, por consiguiente, la opinión de Mariana (publicada en francés en 1625 y antes no divulgada, que sepamos, ni siquiera en ambientes jesuíticos ${ }^{30}$ ). No digo con esto que fuera totalmente errónea; otros humanistas, como el P. Gaspar Sánchez, con 30 años de docencia a sus espaldas, se expresaban en carta al General con parecida franqueza. Pero estos son otros problemas, que deben ser estudiados por sí mismos, y en el contexto español y europeo de la época.

Cervantes se centra en la educación moral, que era substancial e indisimulada en aquel sistema. ¿La condenaba como una muestra del «jesuitismo rampante» que, según Ernest A. Siciliano, había podido observar a su vuelta de la cautividad en 1580 ? $^{31}$. Todos los que se han acercado a la vida de Cervantes se han visto obligados a multiplicar las interrogaciones, si pretenden atenerse a los hechos; las interpretaciones e inferencias pueden ser legítimas e iluminadoras, pero también subjetivas. Cuando los jesuitas de Salamanca en 1610 (o sus escolares, o el pueblo) tomaban al Quijote como "cosa de burla y risa", no se elevaban ciertamente sobre el nivel de aquella sociedad; pero ¿alguien vio más? Quizá, de haber vivido, el "caro y amado" maestro, López de Hoyos...Deducir del panorama social que contempló Berganza, que «we search hard and find very few, if any, members of Spanish society in the Coloquio who have embraced virtue», como afirma Siciliano ${ }^{32}$, deja sin explicar demasiadas cosas. Y apuntar a una posible relación entre el papel de los «benditos padres» y el de la «buena Camacha» ( ¿y por qué no también «el buen cristiano Mahudes»?) con el apoyo de tan polisémico calificativo castellano, abre la puerta a todas las fantasías.

Convengamos en que el hiperbólico elogio no hay que tomarlo ad pedem litterae. ¿No convendría, sin embargo, situarlo previamente en el género literario del elogio o encomium, tal como entonces se practicaba? Un ejemplo, especialmente relacionado con los jesuitas cordobeses. El cordobés Juan de Castilla y Aguayo (o viceversa), que Astrana tiene por coetáneo de Cervantes y por consiguiente posible o probable compañero de aulas ${ }^{33}$, publicó en Salamanca El perfec-

30 En 1614, el General, en su correspondencia con los Superiores de la Provincia de Toledo, se mostraba preocupado por las críticas al gobierno de la Orden, que se sabía hacía el malhumorado historiador; y trataba de impedir su divulgación.

31 The Jesuits in the Quijote and other Essays. Barcelona, 1974, 15.

32 A. c., p. 30. No llega a calificarlos de "rotten», como simplifica BRUCE W. WARDropper, "Cervantes an Education", en Cervantes and the Renaissance, ed. Michael D. McGaho. Easton, Penn, 1980, 187.

33 La amistad con Cervantes consta por el elogio - hay que suponer sinceroque le dedica en el "Canto de Calíope». 
to regidor (1586). En el libro I dedica el c. XI (ff. 55v-59r) a ponderar el "provecho que hace a esta república de Córdoba el colegio de la Compañía de Jesús». El elogio de los métodos de educación moral es demasiado extenso para copiado aquí. Astrana reproduce un fragmento: "no sé qué tiene esta bendita gente, a quien el vulgo llama teatinos, que los mozos que salen disciplinados de sus manos, me parece que sacan diferente espíritu que suelen comúnmente sacar los que se crían debajo de la disciplina de otros preceptores y maestros» ${ }^{34}$. Advierte que "con haber en esta ciudad, antes que vinieran a ella los padres de la Compañía preceptores gramáticos tan hábiles como después acá los han tenido en su colegio, de cien estudiantes no salían entonces cuatro buenos, y ahora de quinientos no aciertan a salir veinte malos». Sigue con otros aspectos, igualmente elogiables, de su ministerio; y no satisfecho, dedica el cap. XII (ff. 59r-64r) a celebrar «quanta utilidad ha sido para la Iglesia católica le bendita Religión de la C. de J.», y lo desarrolla con una contraposición Lutero/Ignacio, y los resultados de la «acomodación» misionera en la India y el Japón (los doce años de presencia en el Perú y México no tienen todavía relevancia).

¿Habrá que leer tal elogio al trasluz, como diría A. Castro? ¿Se sentiría Aguayo obligado/forzado por algo, o disimulaba sus reales sentimientos? Menos sospechoso aún de insinceridad es el insobornable debelador de los estatutos de limpieza de sangre, fr. Agustín Salucio OP, que se expresaba así en un sermón de primero de año en la iglesia del colegio de Córdoba: «Acuerdome yo, cuando en esta ciudad no había sino un preceptor de gramática, la que aprendían los discípulos era tan poca, que apenas había clérigo que pasase de los principios de ella; algunos que tasadamente sabían leer. Ahora en cada rincón hallaréis grandes filósofos, doctos teólogos, llenos de ellos las ciudades y pueblos, las Audiencias y las iglesias y las religiones. [...] De las mejoras de las costumbres, qué os diré, que no veáis?» ${ }^{35}$. Amplificaciones retóricas, sin duda, que nadie tomará

34 Mi subrayado apunta directamente al comienzo de los elogios de Berganza («no sé qué tiene») y Cipión («esa bendita gente»). ¿Casualidad? Astrana (I,24) recoge la opinión de Schevill-Bonilla, en las notas a La Galatea, del posible conocimiento del libro de Aguayo por Cervantes; pero no señalan esta coincidencia, como tampoco Astrana. (Por supuesto que en el texto de Cervantes se debe leer «tan poco", y no "tampoco", como pretende M. L. JAROCKA, o. c., p. 90). A continuación desarrolla Aguayo prolijamente el símil del cultivo de las plantas, lo que para la sobriedad cervantina se condensa en la alusión a las «tiernas varas».

35 J. DE SAnTibáÑEZ, "Historia de la Provincia de Andalucía», I, 1, c. XII. SEBASTIÁN DE COVARRUBIAS, en la voz JESÚs de su Tesoro de la lengua (1611), dedica una columna a los jesuitas con este inciso: "No quiero parecer lisongero, aunque no lo será el que dixere lo que bastara a hacer volumen y libro entero de sus alabanzas. Por otro nombre los llaman teatinos, equivocándose...». 
a la letra; pero indicios de una novedad, que era así apreciada por muchos.

Recordemos por fin que Cervantes dedicó sus Novelas Ejemplares al "gran conde de Lemos», su verdadero mecenas, al que también dedicará las Ocho comedias y ocho entremeses (1615), la segunda parte del Quijote (1615) y Los trabajos de Persiles y Sigismunda, cuatro días antes de morir ${ }^{36}$. Como sabemos, D. Pedro de Castro había sido, junto con sus dos hermanos, alumno del colegio de Monforte, fundado por su tio-abuelo, el arzobispo de Sevilla y cardenal $^{37}$. Es muy posible que esta dedicatoria tenga algo que ver con lo dilatado y hasta redundante del elogio a los jesuitas; pero ¿es verosímil que Cervantes haya aprovechado precisamente esta ocasión para entregarse a un ejercicio tan refinadamente antifrástico? Parece que ni al trasluz, ni de otra manera, cabe tal interpretación.

En resumen, creo con Forcione, que su composición (como conjunto; dejemos la referencia in cauda a la humildad...) en clave de ironía, cuando no de disimulada crítica, sigue siendo «unconvincing» ${ }^{38}$.

JosÉ MARTínEZ-EsCALERA S.J. Universidad Pontificia Comillas

\footnotetext{
En 1555 escribía desde Córdoba el inquisidor Villar al papa Paulo IV: «Es cosa de grande admiración ver la mudança que de costumbres y letras hay en los niños, estudiantes y mançebos desta ciudad y de los lugares comarcanos, que, si dezimos lo que dize el pueblo, desconoçida está Córdoba de lo que solía, y la esperança que de tales principios tenemos, nos promete cosas mayores...» (MHSI, Ignatiana, XII, 565).

36 Eduardo Pardo de Guevara y Valdés, Don Pedro Fernández de Castro VII Conde de Lemos. Santiago, 1997, I, 253-257.

37 En Nápoles promovió la fundación del colegio dedicado al Bto. Francisco Javier; y las relaciones que mantuvo con el General de los jesuitas, Claudio Acquaviva, de la casa napolitana de los duques de Atri, se expresan en términos de mutua confianza y afecto, a pesar de las dificultades que suscitó el P. Fernando de Mendoza, confesor de la condesa madre.

38 «The interpretation is extremely hypothetical, relies to a great extent on extrinsic evidence, i.e. on what is not in Cervantes' text, and places quite an exegetical strain on the few textual elements which it does seize for support» (o. c., 152).
} 